\title{
Meta-analysis of studies on body weight and cholesterol reduction using the chitosan derivative polyglucosamine
} L112

\author{
Cornelli $\mathrm{U}^{1 *}$ and Belcaro $\mathrm{G}^{2}$ \\ ${ }^{1}$ Loyola University School of Medicine, Chicago \\ ${ }^{2}$ Irwin labs University of Chieti, Italy
}

\begin{abstract}
Background: In the Cochrane Database review of 2008 the statement about the relationship between chitosan and body weight (BW) was "Results obtained from bigh quality trials indicate that the effect of chitosan on body weight is minimal and unlikely to be of clinical significance". However, the chitosan family consists of polymers with different chemico-physical characteristics and activity.

Method: Analysis of the literature through PUBMED and EMBASE from 2006 to 2018 concerning controlled clinical trials conducted with a standardized chitosan (polyglucosamine L112 or PG) with MW between 100 and 300 KDa with a deacetylation degree (DD) > 90 \%. Eight articles have been found where PG was compared with a placebo or other treatments to control BW, waist circumference (WC), body mass index (BMI) and four of these were also controlling the total cholesterol (TC) and triglyceride (TG) levels.

Results: The analysis of the 8 articles indicated that: a) at the average dose of $1.7 \mathrm{~g} / \mathrm{day}$ the average BW reduction was $1.44 \mathrm{~kg} / \mathrm{month}$; $\mathrm{b}$ ) the treatment duration was between 2 and 12 months; c) results were achieved in a shorter period of time when the caloric restriction and physical activity were part of the protocol. TC and TG reductions were studied in 4 of the 8 trials showing an average reduction of $9.6 \%$ and $15.3 \%$ respectively. Side effects were detected in about $1 \%$ of the cases.

Conclusion: PG revealed a safe and clinically significant reduction in BW, TC and TG levels compared to placebo or other treatments. Caloric restriction and physical activity accelerate the PG effect.
\end{abstract}

Abbreviations: WC: waist circumference; BMI: body mass index; BW: body weight: CRP: C reactive protein; DD: deacetylation degree; $\mathrm{MET} / \mathrm{h}$ : metabolic equivalents; MW: molecular weight; MR: monthly body weight reduction; Kcal: kilo calories; KDa: kiloDaltons; PG: Polyglucosamine (L112); SEP: substantially equivalent product; TC: total cholesterol; TG: triglycerides.

\section{Introduction}

Chitin, chitosan and chitosan derivatives are an extremely wide chapter of the chemistry that even today represents a challenge due to the nature of these polymers which are the most represented in the biological field [1-3]. The use of chitosan in humans is very common due to the many biological activities of this polymer, starting with the fat binding capacity and followed by the antioxidant and antibacterial/antifungal activities, bleeding control, up to its use as cleaners or stabilizers for wine, beer and many other beverages $[3,4]$.

Chitosans in particular belong to a family of polymers which are derived from the deacethylation of chitin and each may have peculiar characteristics: first of all, the DD, which has to be $>70 \%$, followed by the MW, the solubility, the viscosity and finally by the presence of possible contaminants deriving from the source and the extraction processes. These aspects underline that each chitosan made available by a given producer has its own characteristic and quality profile.
Sources of chitosan are many and very different. Apart from the marine origin (e.g. shrimps, crabs), other sources are now available from vegetables, being extracted from different mushrooms.

These aspects indicate that the chitosans to be used in humans need to comply with at least three important characteristics:

a. Specific MW and DD.

b. Second the degree of polydispersion. This variable is extremely important because even with the same MW the final product can be composed of short polymers (very low MW chitosan or VLMWC with $<30 \mathrm{KDa}$ ) and very large polymers (very high MW chitosan with or VHMWC with $>500 \mathrm{KDa}$ ). It may happen -as in same casethat the final product ends up as a mixture of VLMWC and VHMWC without a sufficient quantity of chains within the requested MW.

c. Third, another fundamental characteristic is the DD which has to be at least $>80 \%$ to ensure an effective lipid binding.

${ }^{\star}$ Correspondence to: Umberto Cornelli, Loyola University School of Medicine, Chicago - 2160 First Ave Maywood IL, Piazza Novelli 520129, Milan, Italy

Key words: chitosan, polyglucosamine, body weight, cholesterol, triglycerides, obesity, caloric restriaction

Received: August 20, 2018; Accepted: August 28, 2018; Published: August 31, 2018 
These three aspects, together with the dimension of the particles, the ashes and the presence of contaminants (proteins, heavy metals, bacteria and other microorganisms) have to be carefully standardized to ensure the appropriate psysico/chemical profile and purity.

For these reasons, each chitosan can have peculiar pharmacological/ clinical activities and one may not generalize, neither for the activity nor for the safety.

Without considering the standardized characteristics that identify any given chitosan, a previous clinical investigations survey conducted in the period up to 2006 -reported in the Cochrane Database of Systematic Review- has been published concerning the activity of chitosan on body weight control [1].

The relevant conclusions of the authors were "there is some evidence that chitosan is more effective than placebo in the short-term treatment of overweight and obesity. However, many of the included trials to date have been of poor quality. Results obtained from high quality trials indicate that the effect of chitosan on body weight is minimal and unlikely to be of clinical significance". However, in the review the physico/chemical characteristics were not considered, and the different products used in clinical trials were taken as a single chitosan.

The present report is related to a specific chitosan, formoline L112 (polyglucosamine or PG) as a Medical Device together with Substantially Equivalent Products (SEP) to PG in the class of food supplements.

\section{Material and Methods}

The method used to retrieve the relevant literature was according to bibliographic search meeting the requirements of MEDDEV 2.7/1 revision 4 .

Search strings were applied to PUBMED and EMBASE, considering only clinical trials from 2006 up to 2018, published after the previous Cochrane analysis which appeared in 2008 (limited to articles published up to 2006) and consisting of papers written in English or with a complete summary in English.

The selection of the investigations to be analyzed was carried out by following six steps:

$1^{\text {st }}$ step: consisted of the isolation of the papers for the period between 2006 and 2018 .

$2^{\text {nd. }}$ step: only those articles containing keywords in the title such as chitosan, polyglucosamine, low molecular weight chitosan and one of the following words: obesity, body weight, overweight, weight loss, nutrition, diet, dietary fibers.

$3^{\text {rd. }}$ step: consisted of the retrieval of the article summaries to determine investigations pertinent to humans only.

$4^{\text {th }}$ step: the selection considered clinical trials following every study design: randomized, placebo-controlled, double blind and registry, paying attention to exclude any articles that feature duplicate information.

$5^{\text {th }}$ step: complete analysis of the investigation conducted in humans and pertinent to chitosan with a declared MW within the range 100 and $300 \mathrm{KDa}$ and deacetylation degree (DD) $>90 \%$.

$6^{\text {th }}$ step: only articles describing the activity on BW, waist circumference (WC) and BMI were considered. Cholesterol levels were also analyzed but as a secondary variable.

\section{Statistical analysis}

The analysis was based on the averages $\pm \mathrm{SD}$ of the published data and the statistical analysis of the single trials was used to determine the PG activity. The correlations among variables were tested using the published average values by performing the density ellipse analysis [5].

\section{Results}

Based upon the criteria specified in the introduction, more than a thousand articles could be retrieved consistent with chitosan and body weight, but among all only the 8 articles showed pertinent characteristics (Table 1). All these articles were retrieved and analyzed for the results.

In total, the number of overweight subjects treated in these 8 articles were 777 ( 429 with PG and 348 as controls) and studied in three countries Italy, Germany and USA. The average age was 49 (in the range from 30 to 60 years) and the side effects due to PG were about $1 \%$ of the cases, represented mainly by temporary constipation (that disappeared by increasing the water intake). Some details of the studies are reported in Table 2.

Duration of treatment ranged from 2 to 12 months and the dosage was from $900 \mathrm{mg} /$ day, up to $2000 \mathrm{mg} /$ day. The lowest dose used was in a study combining PG with a protein surrogate. In all the other investigations, daily dosing was between $1600 \mathrm{mg}$ and $2000 \mathrm{mg} /$ day depending upon the dosage strength per tablet. In Italy, the strength of the tablet was $500 \mathrm{mg}$, whereas in the other countries the tablet weight was $400-500 \mathrm{mg}$, to be taken twice/day before the two main meals for a total of 4 tabs/day.

In one study, the daily dosage was only $900 \mathrm{mg}$ once a day (in association with a protein formula), and in another study, PG was administered as pasta containing $2 \%$ of PG for a total amount of 1.6 g once day.

The average monthly reduction of BW was $1.44 \pm 0.486$ (with a 34 $\%$ coefficient of variation) ranging from $0.77 \mathrm{~kg}$ and $2.23 \mathrm{~kg}$, and the highest value was reached when the caloric restriction and physical activity increase were part of the protocol. The daily caloric reduction (between 300 and $500 \mathrm{kcal} /$ day) allowed for about $16 \%$ greater reduction of $\mathrm{BW}(1.54 \mathrm{~kg}$ vs. $1.41 \mathrm{~kg}$, respectively) but the limited number of studies (4 with caloric restrictions and 4 without) did not allow for any reliable statistical analysis. The same was also shown in the protocols requesting an increase in physical activity. In 5 studies where the increase was between 8 to $21 \mathrm{MET} / \mathrm{h} /$ week, the monthly BW reduction was $35 \%$ more consistent than in the 3 studies with no modifications (1.73 kg vs. $1.38 \mathrm{~kg}$, respectively).

The correlations (based on the calculation for density ellipse) between the BW reduction and the treatment duration in months were find statistically significant $(\mathrm{p}<0.01)$, the same correlating effect was seen with the total amount of PG administered in the period.

Table 1. Selected articles concerning PG L112 and Substantially Equivalent Products (SEP) according to MEDDEV 2.7/1 rev.4 in the period 2006 and 2018

\begin{tabular}{|l|l|l|}
\hline String & $\begin{array}{l}\text { Number } \\
\text { of articles }\end{array}$ & $\begin{array}{l}\text { Number of } \\
\text { Pertinent articles }\end{array}$ \\
\hline Chitosan total & 1210 & 8 \\
\hline Subclass & & \\
\hline Chitosan body weight/diet & 182 & 2 \\
\hline Chitosan obesity & 25 & 2 \\
\hline Chitosan overweight & 25 & 1 \\
\hline Polyglucosamine & 11 & 3 \\
\hline Total & 243 & 8 \\
\hline
\end{tabular}


These correlations should be considered with prudence because both caloric restriction and physical activity were different among the trials. However, despite this limitation, treatment duration can still be considered the most important variable in relation to BW reduction.

Two investigations were conducted for the same period of time (3 months) using the same dosage ( $1.8 \mathrm{~g} /$ day $)$ and the most consistent result (BW $-6,7 \mathrm{~kg}$ ) was found when the treatment protocol included a combination of diet and physical exercise, whereas alone with PG treatment, the diminution was much less (BW $-2.3 \mathrm{~kg}$ ).

A side analysis was also conducted for the total cholesterol and triglycerides. Unfortunately, only 4 studies took these variables into consideration and the results are summarized in table 3 (Table 3 ).

For this set of data, the number of cases was limited to 293 subjects (151 with PG and 142 as control cases).

Despite the differences between baseline data and after treatment, the effects were in every instance statistically significant (Tukey test) and a quite consistent variation of the results was evident. Particularly for total cholesterol the percentage change from baseline ranges from -0.5 and $21.1 \%$ depending upon the experimental condition. The lowest TC reduction was found when PG was added to pasta, whereas the highest was in subject taking tablets. The protocol difference between the two groups was that with pasta the dosage of PG was lower $(1.6 \mathrm{~g} /$ day instead of $1.8 \mathrm{~g} /$ day) and taken once day, whereas with tablets the PG was taken twice/day.

The age of patient was ranging between 30 and 60 years but there was no correlation (ellipse density analysis $p>0.05$ ) between age of the subjects and effect on BW.

\section{Discussion}

The limitation of this meta-analysis was due to the difference among the protocols in terms of dosages, period of administration, caloric restriction and physical training. Furthermore, the authors of the present analysis were also the investigators that conducted most of the clinical trials. The consequence of these limitations is that the results have to be taken with prudence because some bias may not be excluded as it occurs in all the meta-analysis studies.

However, the advantage compared to other similar meta-analysis studies was that the investigators had a first-hand knowledge of most of the patients which provided a more direct evaluation of the results.

In all the trials, the BW reduction was evident and clinically relevant.

This BW reduction effect cannot be considered a common characteristic of all chitosans except for PG which has a peculiar chemico-physical activity (MW, polydispersion and DD) that was well documented.

It has been demonstrated that MW and DD of chitosan are consistent with the fat binding capacity such that high MW polymer loses some fat binding activity and the consequence will be a limited capability for body weight reduction. Polydispersion is also very important because even with the same MW, a wide polydispersion dilutes the polymer solution and reduces the binding capability in relation to the part of the polymers with very high MW (> $300 \mathrm{KDa}$ ) [14]. As a consequence, the batches have to be standardized in order to maintain the same characteristics as much as possible, and this aspect for biological derivatives requires quite a complex know-how.

Every batch of PG is carefully examined for its fat binding capacity with a standardized method [13]. This effect is important because lipid absorption can only be reduced in case the physical form of chitosanlipid aggregates escape the intestinal hydrolysis [15]. This implies that an appropriate emulsion for PG can be stabilized by the presence of organic acids (e.g. ascorbate) as in the present chitosan formula.

The standardized PG has been shown in preclinical studies to reduce the absorption of labelled (C14) oleic acid in Göttingen minipigs, to reduce the $\mathrm{BW}$ and increase fat excretion in rats and to increase the acetate excretion in rats [16-18]. This last aspect is considered important

Table 2. Summary of the clinical investigations according to the main variables BW, AC and BMI reported as differences (diff) from the baseline values $\pm \mathrm{SD}$; [N] number of subjects

\begin{tabular}{|c|c|c|c|c|c|c|c|c|c|c|}
\hline Reference & $\begin{array}{c}\text { Duration } \\
\text { months }\end{array}$ & $\begin{array}{l}\text { Dose } \\
\text { g/day }\end{array}$ & $\begin{array}{c}\text { Diet } \\
\text { Kcal/day }\end{array}$ & $\begin{array}{l}\text { Phys act } \\
\text { MET-h/W }\end{array}$ & $\begin{array}{c}\text { BW }^{\mathrm{d}} \\
\text { diff }[\mathrm{kg}] \\
\text { PG }[\mathrm{N}]\end{array}$ & $\begin{array}{l}\text { BW diff }[\mathrm{kg}] \\
\text { Controls }[\mathrm{N}]\end{array}$ & $\begin{array}{c}\mathrm{AC}^{\mathrm{e}} \\
\operatorname{diff}[\mathrm{cm}] \\
\text { PG }\end{array}$ & $\begin{array}{c}\mathrm{AC} \\
\operatorname{diff}[\mathrm{cm}] \\
\text { Controls }\end{array}$ & $\begin{array}{c}\text { BMI }^{\mathrm{f}} \\
\operatorname{diff}\left[\mathbf{k g} / \mathrm{m}^{2}\right] \\
\text { PG }\end{array}$ & $\begin{array}{c}\text { BMI } \\
\operatorname{diff}\left[\mathrm{kg} / \mathrm{m}^{2}\right] \\
\text { Controls }\end{array}$ \\
\hline 6 & 3 & 0.9 & $-500^{\mathrm{b}}$ & No & $-5.46[52] \pm 3.83$ & $-4.72[54] \pm 3.88$ & $-5.83 \pm 3.84$ & $-5.59 \pm 3.82$ & $-1.76 \pm 1.20$ & $-1.5 \pm 1.17$ \\
\hline 7 & $3^{\mathrm{a}}$ & 1.8 & -500 & 21 & $-6.7[28] \pm 3.14$ & $-4.8[28] \pm 2.24$ & $-8.3 \pm 4.42$ & $-6.1 \pm 3.43$ & $-2.3 \pm 1.09$ & $-1.7 \pm 0.86$ \\
\hline 8 & 6 & 1.8 & -500 & 7 & $-6.5[45] \pm 3.89$ & $-4.3[42] \pm 3.12$ & $-9.1 \pm 6.83$ & $-7.4 \pm 7.66$ & $-2.4 \pm 1.40$ & $-1.6 \pm 1.18$ \\
\hline 9 & 12 & 1.8 & $-300^{\mathrm{g}}$ & 9 & $-12.2[49] \pm 3.06$ & $-8.0[48] \pm 2.81$ & $-14.1 \pm 3.19$ & $-10.2 \pm 3.01$ & $-4.3 \pm 1.12$ & $-2.8 \pm 0.91$ \\
\hline 10 & $2^{\mathrm{h}}$ & 1.6 & No & 8 & $-2.6[22] \pm 3.83$ & $-1.0[12] \pm 0.99$ & $-6.1 \pm 3.36$ & $-2.9 \pm 0.50$ & $-0.9 \pm 0.61$ & $-0.4 \pm 0.37$ \\
\hline 11 & 3 & 2.0 & No & No & $-4.6[191] \pm 1.81$ & $-1.2[122] \pm 0.56$ & $-5.1 \pm 2.33$ & $-4.2 \pm 2.31$ & $-1.4 \pm 0.60$ & $-0.4 \pm 0.30$ \\
\hline 12 & 3 & 1.8 & No & No & $-2.3[14] \pm 1.38$ & $-1.9[14] \pm 0.95$ & $-1.9 \pm 3.37$ & $-1.6 \pm 1.08$ & $-0.8 \pm 0.49$ & $-0.6 \pm 0.33$ \\
\hline 13 & 4 & 2.0 & No & $8-12$ & $-6.9[28] \pm 1.87$ & $-3.0[28] \pm 1.61$ & $-7.3 \pm 2.49$ & $-3.1 \pm 4.21$ & $-2.3 \pm 0.84$ & $-1.0 \pm 0.53$ \\
\hline
\end{tabular}

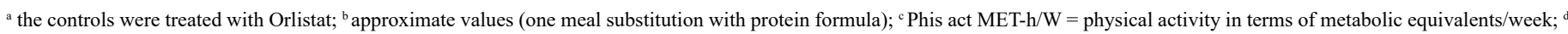
Body Weight; ${ }^{\mathrm{e}}$ Waist circumference; ${ }^{\mathrm{f}}$ Body Mass Index; ${ }_{\mathrm{g}}^{\mathrm{g}}$ approximate value corresponding to $10 \%$ reduction; ${ }^{\mathrm{h}} \mathrm{PG}$ was added to pasta and controls were eating normal pasta

Table 3. Summary of the clinical investigations according to the variables $\mathrm{N}-$ number of subjects, Total cholesterol (TC) and triglycerides (TG) reported as \% reduction \pm SD from baseline; $[\mathrm{N}]$ number of subjects

\begin{tabular}{|c|c|c|c|c|c|c|c|c|}
\hline Reference & $\begin{array}{c}\text { Duration } \\
\text { months }\end{array}$ & $\begin{array}{l}\text { Dosage } \\
\text { g/day }\end{array}$ & $\begin{array}{c}\text { Diet } \\
\text { Kcal/day }\end{array}$ & $\begin{array}{l}\text { Phys act } \\
\text { MET-h/W }\end{array}$ & $\begin{array}{c}\text { TC } \\
\text { \% reduction } \\
\text { PG }[N]\end{array}$ & $\begin{array}{c}\mathrm{TC} \\
\% \text { reduction } \\
\text { Controls [N] }\end{array}$ & $\begin{array}{c}\text { TG } \\
\text { \% reduction } \\
\text { PG }[N]\end{array}$ & $\begin{array}{c}\text { TG } \\
\% \text { reduction } \\
\text { Controls }[\mathrm{N}]\end{array}$ \\
\hline 6 & 3 & 0.9 & $-500^{b}$ & No & $-7.4[52] \pm 0.75$ & $-1.1[54] \pm 0.78$ & $-13.8[52] \pm 0.78$ & $-11.0[54] \pm 1.32$ \\
\hline 9 & 12 & 1.8 & $-300^{c}$ & 9 & $-9.6[49] \pm 0.43$ & $-4.6[48] \pm 0.29$ & $-17.3[49] \pm 1.73$ & $-12.2[48] \pm 1,18$ \\
\hline 10 & $2^{\mathrm{h}}$ & 1.6 & No & 8 & $-0.5[22] \pm 0.10$ & $-4.7[12] \pm 0.69$ & $-6.7[22] \pm 3.96$ & $-19.8[12] \pm 12.87$ \\
\hline 13 & 4 & 2.0 & No & $8-12$ & $-21.1[28] \pm 1.90$ & $-8.1[28] \pm 0.65$ & $-23.3[28] \pm 3,22$ & $-16.6[28] \pm 2.16$ \\
\hline
\end{tabular}

${ }^{\mathrm{b}}$ approximate values (one meal substitution with protein formula)

${ }^{c}$ Phis act MET-h/week = physical activity in terms of metabolic equivalents/week; ${ }^{\text {h }}$ PG was added to pasta and controls were eating normal pasta. 
for the satiety, since acetate was shown to stimulate the grehlin secretion inducing hyperphagia and related sequelae [19].

In general, for many subjects under treatment we realized that the compliance for the caloric restriction was very good; this aspect could be due to the elimination of acetate but also by the increase of satiety by the PG binding with fat, water and other negatively charged components of foods $[4,18,19,20]$.

Another important aspect related to PG was the reduction of CRP (C-reactive protein) that testifies an anti inflammatory and antioxidant activity [9].

Even when caloric restriction or physical activity increase were not requested (free-living conditions), some body weight reduction was evident and clinically significant $[11,12]$. However, the effects on BW and WC were achieved more rapidly and were more consistent in subjects who followed caloric restriction and increased physical activity.

Cholesterol reduction is a well-known and documented effect of chitosans such that the claim is accepted by the EU. This characteristic is common in most chitosans and was also clinically relevant for PG.

\section{Conclusion}

In conclusion, from the analysis of 8 clinical trials conducted with a controlled methodology, PG showed at least three clinically relevant activities, the reduction in body weight, cholesterol and triglyceride levels.

Side effects in general were estimated around $1 \%$ of the treated cases.

\section{Acknowledgement}

Statistical analysis was conducted by a separate institution and we thank Prof. Martino Recchia for his contribution to the analysis.

\section{Author contribution}

Umberto Cornelli and Giovanni Belcaro equally contributed to the analysis of the literature. Umberto Cornelli wrote the text.

\section{Conflict of interest}

Umberto Cornelli and Giovanni Belcaro were author or co-author of most of the clinical trials with PG.

\section{References}

1. Jull AB, Ni Mhurchu C, Bennett DA, Dunshea-Mooij CA, Rodgers A (2008) Chitosan for overweight or obesity. Cochrane Database Syst Rev 3: CD003892. [Crossref]
2. Bellich B, D'Agostino I, Semeraro S, Gamini A, Cesàro A, et al. (2016) “The Good, the Bad and the Ugly" of Chitosans. Mar Drugs 14. [Crossref]

3. Cheung RC, Ng TB, Wong JH, Chan WY (2015) Chitosan: An Update on Potentia Biomedical and Pharmaceutical Applications. Mar Drugs 13: 5156-5186. [Crossref]

4. Younes I, Rinaudo M (2015) Chitin and chitosan preparation from marine sources. Structure, properties and applications. Mar Drugs 13: 1133-1174. [Crossref]

5. Wonnacott TH, Wonnacott RJ, editor (1981) Regression: a second course in statistics Lund, Sweden: John Wiley and Sons.

6. Willerrs J, Plötz SC, Hahn A (2012) The combination of high-protein formula diet and polyglucosamine decreases body weight and parameters of glucose and lipid metabolism in overweight and obese men and women. Europ J Food Res \& Review 2: 29-45.

7. Stoll M, Bitterlich N, Cornelli U (2017) Randomized, double blind, clinical investigation to compare orlistat $60 \mathrm{mg}$ and customized polyglucosamine, two treatment methods for the management of overweight and obesity. BMC Obesity 4: 4 .

8. Pokhis K, Bitterlich N, Cornelli U, Cassano G (2015) Efficacy of polyglucosamine for weight loss- confirmed in a randomized double-blind, placebo-controlled clinical investigation. BMC Obesity 2: 25.

9. Cornelli U, Belcaro G, Recchia M, D'Orazio N (2017) Long-Term Treatment of Overweight and Obesity with Polyglucosamine (PG L112): Randomized Study Compared with Placebo in Subjects after Caloric Restriction. Curr Dev Nutr 1: e000919. [Crossref]

10. Cornelli U, Silveri S, Belcaro G (2008) Pasta intake to reduce body weight. Min Cardioang 56: 77-84.

11. Cornelli U, Milani M, Perra A (2006) The use of polyglucosamine in the metabolic syndrome control. Med Biol 4: 45-54.

12. Cornelli U (2007) ARD Cholesterol ${ }^{\circledR}$ and HDL increase. Med Biol 3: 13-20.

13. Cornelli U, Belcaro G, Cesarone MR, Cornelli M (2008) Use of polyglucosamine and physical activity to reduce body weight and dyslipidemia in moderately overweight subjects. Min Cardioang 56: 71-78.

14. Raisch K, Rockway S (2005) Validation of a quantitative fat binding assay for PG (L112). Nutrition June 2005 Geneva - Switzerland.

15. Muzzarelli RA (1997) Human enzymatic activities related to the therapeutic administration of chitin derivatives. Cell Mol Life Sci 53: 131-140.

16. Cnubben NPH, Tel SL, Hemmes MA (2016) A single oral dose of polyglucosamine influences the bioavailability of [9-14C] oleic acid in adult female Göttingen minipigs. BMC Obesity 3:18.

17. Bondiolotti G, Bareggi SR, Frega NG, Strabioli S, Cornelli U (2007) Activity of two different polyglucosamines, L112 and FF4, on body weight in male rats. Eur J Pharmacol 567: 155-158. [Crossref]

18. Bondiolotti G, Cornelli U, Strabbioli RS (2011) Effect of polyglucosamine on body weight of male rats. Food Chem 124. 978-982.

19. Perry RJ, Peng L, Barry NA, Cline GW (2016) Acetate mediates a microbiome-brain$\hat{\mathrm{I}}^{2}$-cell axis to promote metabolic syndrome. Nature 534: 213-217. [Crossref]

20. Heber D (2003) Herbal preparations for obesity: are they useful? Prim Care 30: 441 463. [Crossref]

Copyright: (C2018 Cornelli U. This is an open-access article distributed under the terms of the Creative Commons Attribution License, which permits unrestricted use, distribution, and reproduction in any medium, provided the original author and source are credited. 